\title{
Article \\ Atomic Concealment Due to Loss of Coherence of the Incident Beam of Projectiles in Collision Processes
}

\author{
Raúl O. Barrachina ${ }^{1, * \mathbb{C}}$, Francisco Navarrete $\left.{ }^{2} \mathbb{(}\right)$ and Marcelo F. Ciappina ${ }^{3,4}(\mathbb{D}$ \\ 1 Centro Atómico Bariloche, Comisión Nacional de Energía Atómica, CNEA, Instituto Balseiro, \\ CNEA and Universidad Nacional de Cuyo, Consejo Nacional de Investigaciones Científicas y Técnicas, \\ CONICET, Av. Bustillo 9500, 8400 Bariloche, Argentina \\ 2 Institute of Physics, University of Rostock, 18051 Rostock, Germany; francisco.navarrete@uni-rostock.de \\ 3 Physics Program, Guangdong Technion-Israel Institute of Technology, Shantou 515063, China; \\ marcelo.ciappina@gtiit.edu.cn \\ 4 Technion-Israel Institute of Technology, Haifa 32000, Israel \\ * Correspondence: barra@cab.cnea.gov.ar
}

check for

updates

Citation: Barrachina, R.O.;

Navarrete, F.; Ciappina, M.F. Atomic Concealment Due to Loss of Coherence of the Incident Beam of Projectiles in Collision Processes. Atoms 2021, 9, 5. https://doi.org/10.3390/atoms9010005

Received: 30 November 2020

Accepted: 12 January 2021

Published: 15 January 2021

Publisher's Note: MDPI stays neutral with regard to jurisdictional clai$\mathrm{ms}$ in published maps and institutional affiliations.

Copyright: $\odot 2021$ by the authors. Licensee MDPI, Basel, Switzerland. This article is an open access article distributed under the terms and conditions of the Creative Commons Attribution (CC BY) license (https:// creativecommons.org/licenses/by/ $4.0 /)$.

\begin{abstract}
In the study of collision processes, a series of conditions is usually assumed. One of them is that the beam of projectiles is coherent in lengths greater than those of the targets against which it strikes. However, recent experimental results and theoretical analyzes have shown that this assumption can not only fail, but that it is possible to manipulate the coherence length experimentally to go from a coherent situation to an incoherent one. The most conspicuous and studied manifestation of such loss of coherence is the disappearance of interference effects. However, in the present work we show that a strong decrease can also occur in the magnitude of the cross section, not only differential but also total, due to an atomic concealment effect.
\end{abstract}

Keywords: collision; coherence; contextuality

\section{Introduction}

For more than a century, since the first measurements made by Rutherford's team in Manchester (see e.g., [1]), most of all studies carried out on collision processes in their different variants, nuclear, atomic, molecular and optical, have assumed a strong independence from the context established by the experimental setup. For example, although that first modern collision experiment [1] consisted of the scattering of alpha particles by gold foil, the velocity of the projectiles was fast enough that the probability of a double collision could be assumed to be negligible.

In the same way, since the distance between the source of projectiles and the target and the width of the collimators are of macroscopic size, it was assumed, and even demonstrated (e.g., [2]), that though the system is in a mixed state, it is possible to describe each individual collision in terms of wave functions rather than quantum density operators. This is, in fact, the basis of the stationary scattering theory [2].

However, recent experiments and subsequent theoretical analyzes (see e.g., [3,4], respectively, and references therein) have challenged these basic assumptions. In particular, the importance of the concept of quantum coherence has been highlighted. Furthermore, it was shown that it can be modified by changing the experimental setup. This loss of coherence is manifested mainly in a disappearance of interference spectra and other associated quantum effects.

The purpose of this work is to show that there is an additional effect, unrelated to the previous ones, that can affect even the total cross sections, and that due to its important magnitude, should be easily observable both experimentally and theoretically.

In the next section we review some basic concepts of the definition of cross section and its assumptions, such as the single scattering condition, which is discussed in Section 3. 
In Section 4 we analyze the usual foundations of the Stationary Scattering Theory, and in Section 5 we show their failures. In Section 6 we show how a loss of coherence of the incident beam can lead to a fading of interference effects. Finally, in Section 7 we introduce the concept of atomic concealment and its influence on the magnitude of differential and total cross sections.

\section{Definition of the Cross Section}

Scattering theory, which is used as a standard to study all kinds of collision processes, incorporates a series of assumptions that are taken for granted. We see this from the very beginning in the definition of the concept of cross section.

When we consider a flow $J$ of projectiles that impinges on a target formed by $N$ particles (e.g., nuclei, atoms, molecules); the number $I$ of events detected per unit time is not adequate to describe the collision process itself, since being proportional to $J$ and $N$, it depends on the specific characteristics of the experiment. For this reason, the cross-section is defined as:

$$
\sigma=I / J N \text {, }
$$

which is clearly independent of the incident beam flux and the number of particles on the target [5] (p. 110).

It is important to note that in the above equation both $\sigma$ and $I$ can refer to multichannel collision processes of different complexity, such as excitation, charge exchange, ionization, fragmentation, fission, etc. In order not to complicate the notation, the dependence on the corresponding variables, whether discrete or continuous, have not been explicitly incorporated.

\section{Single Scattering Condition}

Coming back to Equation (1), for this definition of a cross section to be valid, the single scattering condition (SSC) must be met. It assumes that the collisions between the particles in the incoming beam and the target occur one by one, individually, and that the probability that a projectile hit more than a target particle is insignificant. If this condition is not fulfilled, the assumed linearity of $I$ with $J$ and $N$ would no longer be valid and this would produce a modification in the cross section, not only in shape, but also in magnitude.

Obviously, this condition is not generally verified in collisions with solid targets or surfaces (with exceptions as e.g., [1]), but it certainly is valid in the case of sufficiently dilute gases.

\section{Stationary Scattering Theory}

The other two basic assumptions of the scattering theory are somewhat more subtle, but of enormous importance. Perhaps, their origin can be traced to the standard reference textbook by Leonard Schiff [5], where he indicates that "the bombarding particles [... ] are collimated by diaphragms [... ]. Such a collimated beam is not an infinite plane wave [... ]. [However] at the point of observation, $\mathrm{P}$, only [a] term is present, and it is essentially the same as that which appears [ . . by ] the idealization implicit in assuming an infinite plane wave [... ]" [5].

As a reviewer of the book wrote in 1950, this idea, as others in the book, was not new and could not be attributed to Schiff himself [6]. Perhaps it is not so well-known that this argument can be traced back to a famous discussion between de Broglie and Pauli that occurred at the fifth Solvay Conference in Brussels, during which de Broglie said that "One can speak of the beam [ ... ] only if [ .. ] the incident wave is laterally limited, because otherwise [the scattered wave] would be bathed in the incident wave. [ ... ] One must assume the wave $\psi$ to be limited laterally in configuration space" [7].

We still use this infinite plane wave model. In our calculations we might take care of the correct boundary conditions and other similar subtleties, but without questioning that fundamental and basic assumption. 
As far as we know, the validity of this ansatz was investigated for the first time by Wichmann in 1965 [8]. Notably, this article remains little known, having been cited very few times in more than fifty years. However, his analysis was picked up without citation in some few textbooks (e.g., [9]), more notably by John Robert Taylor in 1972 [2].

It is not our intention to review this demonstration in all details. Let us just say that Wichmann and Taylor's demonstrations are based on two basic assumptions. One is that the aperture $D$ of the collimator is macroscopic and, therefore, much larger than any other length $a$ associated to the scattering event,

$$
D \gg a \text {. }
$$

This same argument might be applied to the distance $L$, travelled by the projectiles before hitting the target; namely,

$$
L \gg a \text {. }
$$

These two assumptions are of the uttermost importance, since by the so-called asymptotic condition and the use of Möller operators, the description of the scattering process becomes stationary [2], endorsing the standard stationary approach with plane waves.

\section{Validity of the Stationary Scattering Theory}

The validity of the Stationary Scattering Theory, rooted in assumptions (2) and (3), has been recently challenged by a series of experiments (see, e.g., [3] and references therein), where the length $L$ between the collimator and the target and/or the aperture $D$ of the collimator are modified. It is important to point out that these techniques should not be mistakenly understood as a deliberate worsening of the experimental uncertainties. Instead of being related to the detection stage, they represent a controlled preparation of the initial set-up.

In view of these experimental results, we have recently been critically reviewing [10-12] the demonstrations by Wichmann [8] and Taylor [2], by representing the projectiles beam as an incoherent mixture of wave packets of linear momentum $p$ (i.e., de Broglie wavelength, $\lambda=2 \pi \hbar / p)$, which differ in their impact parameter. These impact parameters are limited to certain region of width $D$. This starting point implies the incorporation of classical and quantum statistics within a density operator formalism. The main result of this analysis is given by the so-called coherence length:

$$
\ell=\lambda L / \pi D,
$$

which is a quantum-mechanical equivalent to what is known in Optics as the van CittertZernike $[13,14]$ theorem. Now, the condition for the validity of the "idealization implicit in assuming an infinite plane wave" reads [11,12]:

$$
\ell \gg a \text {. }
$$

Thus, the macroscopic lengths $L$ and $D$ are intertwined in such a way that they cannot be compared solely with the atomic size length $a$, as Wichmann and Taylor did. In fact, both $L$ and $D$ can be (and are) macroscopic, and still not verify the previous condition (5).

\section{Coherence Length and Interference}

The result expressed in Equation (5) has relevant consequences insofar as, even when the lengths $L$ and $D$ are macroscopic, it may happen that the coherence length $\ell$ is not much greater than characteristic lengths $a$ such as, for example, the nuclear radius, the atomic radius or the internuclear distances in a molecule, depending on the collision process under study. If so, the basic assumption that justifies the validity of the stationary collision theory is no longer legitimate, and this may lead to the appearance (or disappearance) of observable effects. 
It should be noted that, although a generalization of the collision theory that is valid for coherence lengths $\ell$ of the order or less than the characteristic distance $a$ is not yet available, the various approaches attempted to date have shown that an effective understanding of the effects that usually occur in such circumstances is possible, achieving a good agreement with the available experiments [3,4].

Let us discuss these effects by means of a quite simple model, usually employed with great success in the description of collision processes with molecular targets. In the framework of a linear combination of atomic orbitals (LCAO) approach, we can approximate the transition matrix element as a sum of partial amplitudes $A_{i}$ corresponding to each of the $n$ atoms in the molecule:

$$
\sigma=\left|\sum_{i=1}^{n} A_{i}\right|^{2}=\sum_{i=1}^{n}\left|A_{i}\right|^{2}+\sum_{i \neq j} A_{i} A_{j}{ }^{*} .
$$

Naturally, this model has serious limitations, one of them being the description of conjugated systems. However, it represents a good case study for the present discussion, and only in that sense are we using it here.

One of the most notable effects of this result is that, given the appropriate conditions, that is, if the de Broglie wavelength of the incoming beam is comparable with the internuclear distances in the molecule, then an interference pattern will emerge as the result of the crossed terms of the previous development.

However, if $\ell$ is smaller than these distances, then the projectile beam will not coherently illuminate the whole molecule. Only the first summation of Equation (6) remains, and the interference pattern disappears.

This effect can be clearly seen in the following figure, where the cross section for the ionization of hydrogen molecules by the impact of $75 \mathrm{keV}$ protons doubly differential in the scattering angle $\theta$ and the energy loss $\varepsilon$ of the projectiles, shows an interference pattern for $L=50 \mathrm{~cm}$, which disappears for $L=6.5 \mathrm{~cm}$. In both cases, $D=0.15 \mathrm{~mm}$ and $\varepsilon=30 \mathrm{eV}$.

This transition from a completely coherent situation to an incoherent one is observed both in the experimental [15] and theoretical [10] results. For the calculation, we employed the molecular version of the three-body distorted-wave-eikonal initial-state (M3DW-EIS) approach developed by Chowdhury et al. [16]. A full and comprehensive description of this model can be found in the aforementioned article, where it is shown to correctly account for the two-center effects inherent to the collision of a $75 \mathrm{keV}$ proton with a $\mathrm{H}_{2}$ molecule. For the description of the effects produced by the coherence length $\ell$, we employed the angle convolution described in Ref. [10]. We refer the reader to this article for further details of how this technique allows for the incorporation of the coherence effects in the description of the scattering process.

The explanation for the effect shown in Figure 1 lies in the very definition of the coherence length, Equation (5), which changes from $\ell \approx 110 \AA$ to $\ell \approx 15 \AA$ for $L=50 \mathrm{~cm}$ and $L=6.5 \mathrm{~cm}$, respectively. In the first case, the coherence length is larger than the internuclear distance, $a=74 \AA$, and the whole molecule is coherently illuminated, while in the second it is not. 


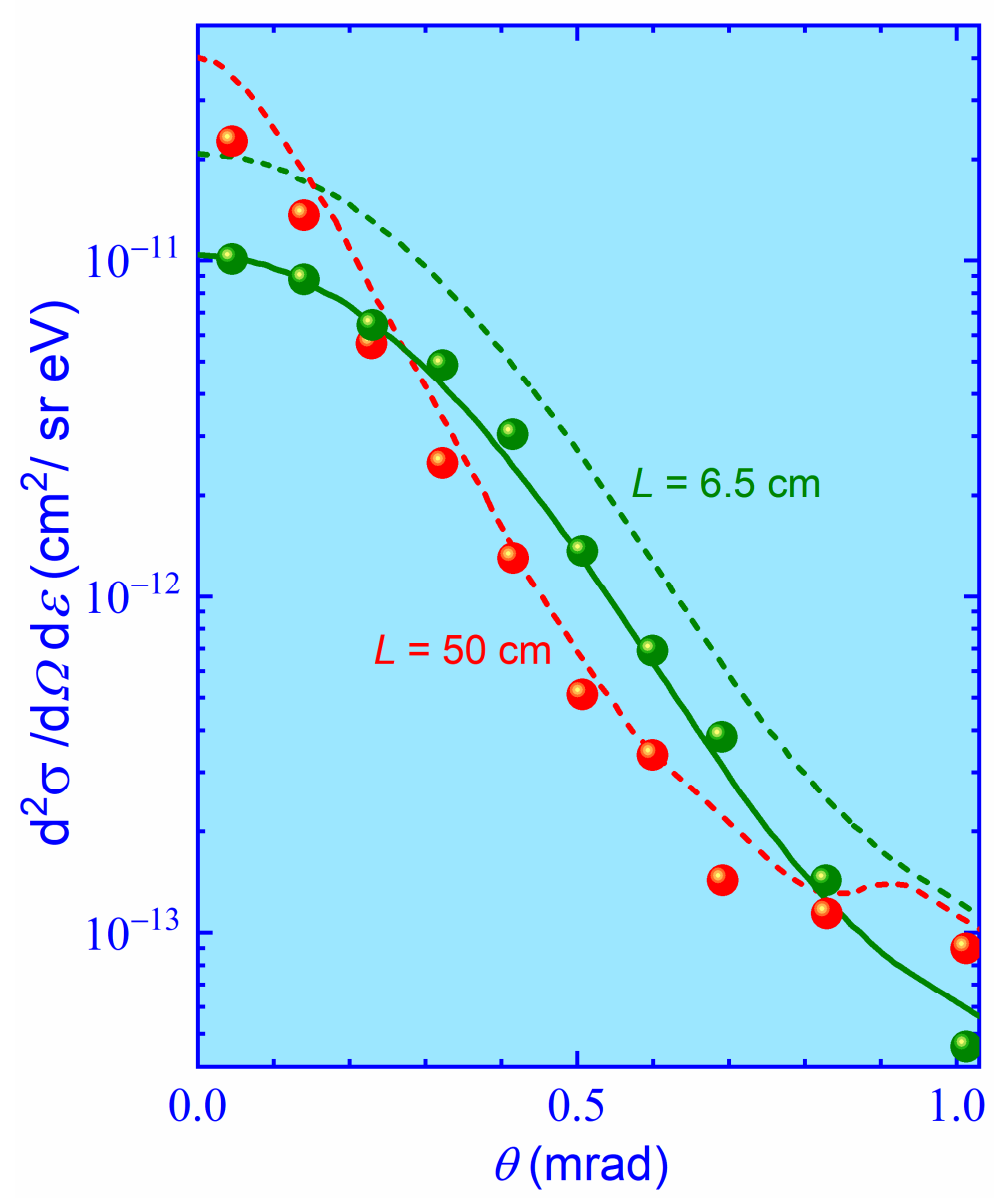

Figure 1. Doubly differential cross section for the ionization of $\mathrm{H}_{2}$ by the impact of $75 \mathrm{keV}$ protons as a function of the scattering angle $\theta$ and the energy loss $\varepsilon$ of the projectiles. The energy loss is $30 \mathrm{eV}$. The experimental (circles) [15] and theoretical (dashed lines) [10] results are compared, for $L=6.5 \mathrm{~cm}$ (green) and $50 \mathrm{~cm}$ (red). In both cases, $D=0.15 \mathrm{~mm}$. The solid green line incorporates the atomic concealment effect into the theoretical result (dashed green line) for $L=6.5 \mathrm{~cm}$, as described in Section 7.

\section{Atomic Concealment}

In Figure 1 the presence of another effect produced by the loss of coherence of the incident beam should be evident. In fact, we see that the calculation for $L=6.5 \mathrm{~cm}$ is overestimating the experimental results by a factor two. In this article we propose that this result is due to an effect that had not been properly explained until now.

Returning to the pilot LCAO model characterized by Equation (6), we can infer that when a projectile collides with one of the atoms of the molecule, it does so with that atom and nothing else. In other words, it cannot collide with any of the others. It would be like illuminating a series of objects with a flashlight. If its angular aperture is large enough, then we will see all objects simultaneously. But if that aperture is less than the distance between the objects, we will only be able to see one of them at a time, with the rest remaining hidden in the shadows.

The same is happening in this situation, and therefore the sum of Equation (6) must be replaced by an average, namely:

$$
\sigma \approx \frac{1}{n} \sum_{i=1}^{n}\left|A_{i}\right|^{2}
$$


Thus, we see that in Figure 1, the theoretical calculation represented by the dashed line for the incoherent case must be divided by a factor $n=2$ as shown with the full (green) line, achieving a perfect fit with the experimental results.

If we compare this result with the single scattering condition described in Section 3, we see that the physics of the problem is similar. In the framework of the ion-molecule collision that we are analyzing as a pilot case, as well as in the framework of the SSC where each projectile can collide with one and only one molecule, we now incorporate the condition that, if the coherence length is small enough, it can only collide with one, and only one, of the atoms of each molecule.

This result implies that the loss of coherence can affect not only differential cross sections, but also the total ones. In fact, integrating the dashed lines in Figure 2 as a function of the angle $\theta$, we obtain the same cross section:

$$
d^{2} \sigma / d \varepsilon=4.0 \times 10^{-14} \mathrm{~cm}^{2} / \mathrm{eV}
$$

for $L=50 \mathrm{~cm}$ and $L=6.5 \mathrm{~cm}$. But now, the incorporation of the atomic concealment effect reduces this latter cross section by a factor of 2 for $L=6.5 \mathrm{~cm}$, i.e.,

$$
d^{2} \sigma / d \varepsilon=2.0 \times 10^{-14} \mathrm{~cm}^{2} / \mathrm{eV} .
$$

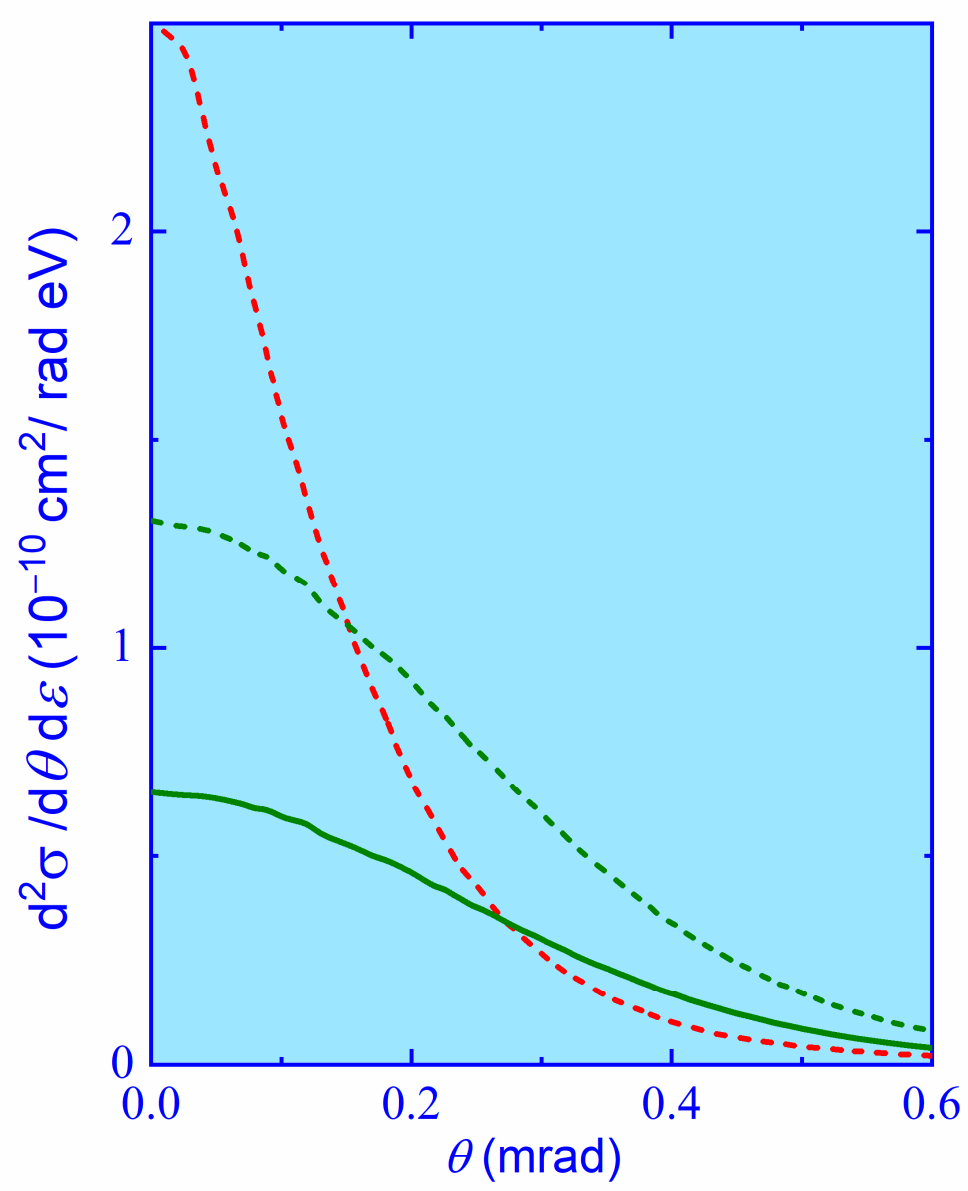

Figure 2. Doubly differential cross section for the ionization of $\mathrm{H}_{2}$ by the impact of $75 \mathrm{keV}$ protons as a function of the scattering angle $\theta$ and the energy loss $\varepsilon$ of the projectiles. The energy loss is $30 \mathrm{eV}$. The theoretical [10] results are compared, for $L=50 \mathrm{~cm}$ (dashed red line) and $6.5 \mathrm{~cm}$, with (solid green line) and without (dashed green line) the atomic concealment effect. In both cases, $D=0.15 \mathrm{~mm}$. Note that the logarithmic scale of the vertical axis in Figure 1 has been changed to a linear one. 


\section{Conclusions}

In this work we showed that, in addition to the disappearance of the interference spectra, the loss of spatial coherence of the incident beam in any given collision process, also produces a significant change in the magnitude of the cross section.

The second aspect that we must emphasize is that conditions (2) and (3) can no longer be considered as sufficient for the validity of the usual theory of stationary collisions. Therefore, it would be necessary to verify in the theoretical developments of collision processes if the condition (4) involving the length of coherence is fulfilled, in order to be able to ensure whether or not the said theory applies to the experimental set-up being considered.

Despite the efforts made by different authors $[4,10,17-19]$ in recent years, there is still no generalization of the stationary collision theory that is valid for every value of the coherence length. Thus, we can only describe what happens at the limits when $\ell$ is much greater or much less than $a$, as shown in Equations (6) and (7), respectively. For intermediate situations, we can only infer that as $\ell$ decreases, the magnitude of the cross section will decrease with a multiplicative factor that will vary from 1 to $1 / n$.

This shows that it is essential to develop such a generalization of the standard theory to be able to achieve a complete understanding of collision processes in all the variety of conditions that can occur.

Finally, it is important to point out that, despite an exhaustive search in the existing literature, it was not possible to find other ion-molecules experiments like the one analyzed here. Future new experiments with variable coherence lengths would be much welcomed to validate or question the conclusions made in this article.

Author Contributions: Conceptualization, R.O.B.; F.N.; M.F.C.; investigation, R.O.B.; F.N.; M.F.C.; writing-original draft preparation, R.O.B.; F.N.; M.F.C.; writing-review and editing, R.O.B.; F.N.; M.F.C. All the authors contributed equally to this article. All authors have read and agreed to the published version of the manuscript.

Funding: This research received no external funding.

Institutional Review Board Statement: Not applicable.

Informed Consent Statement: Not applicable.

Data Availability Statement: Data sharing not applicable.

Conflicts of Interest: The authors declare no conflict of interest. The funders had no role in the design of the study; in the collection, analyses, or interpretation of data; in the writing of the manuscript, or in the decision to publish the results.

\section{References}

1. Geiger, H.; Marsden, E. The Laws of Deflexion of Alpha Particules through Large Angles. Phil. Mag. S. 6 1913, 25, 604-623. [CrossRef]

2. Taylor, J.R. Scattering Theory: The Quantum Theory of Nonrelativistic Collisions; Wiley: New York, NY, USA, 1972.

3. Schulz, M. The Role of Projectile Coherence in the Few-Body Dynamics of Simple Atomic Systems. Adv. Atoms. Mol. Opt. Phys. 2017, 66, 507-543.

4. Barrachina, R.O.; Navarrete, F.; Ciappina, M.F.; Schulz, M. Coherence and contextuality in scattering experiments. In Ion-Atom Collisions: The Few-Body Problem in Dynamic Systems; Schulz, M., Ed.; De Gruyter: Berlin, Germany, 2019; pp. 61-80.

5. Schiff, L.I. Quantum Mechanics, 1st ed.; McGraw-Hill: New York, NY, USA, 1949; p. 116.

6. Seitz, F. LI Schiff, Quantum Mechanics. Bull. Am. Math. Soc. 1950, 56, 191-192. [CrossRef]

7. Bacciagaluppi, G.; Valentini, A. Quantum Theory at the Crossroads. In Reconsidering the 1927 Solvay Conference; Cambridge University Press: Cambridge, UK, 2009.

8. Wichmann, E.H. Scattering of Wave Packets. Am. J. Phys. 1965, 33, 20-31. [CrossRef]

9. Gottfried, K.; Yan, T.-M. Quantum Mechanics: Fundamentals, 2nd ed.; Springer: New York, NY, USA, 2003.

10. Sarkadi, L.; Fabre, I.; Navarrete, F.; Barrachina, R.O. Loss of wave-packet coherence in ion-atom collisions. Phys. Rev. A 2016, 93, 032702. [CrossRef]

11. Fabre, I.; Navarrete, F.; Sarkadi, L.; Barrachina, R.O. Free Evolution of an Incoherent Mixture of States: A quantum mechanical approach to the Van Cittert-Zernike Theorem. Eur. J. Phys. 2018, 39, 015401. [CrossRef] 
12. Barrachina, R.O.; Navarrete, F.; Ciappina, M.F. Free evolution of a mixture of quantum states with classical space and momentum uncertainties. Eur. J. Phys. 2019, 40, 065402. [CrossRef]

13. Van Cittert, P.H. Die Wahrscheinliche Schwingungsverteilung in Einer von Einer Lichtquelle Direkt Oder Mittels Einer Linse Beleuchteten Ebene. Physica 1934, 1, 201. [CrossRef]

14. Zernike, F. The concept of degree of coherence and its application to optical problems. Physica 1938, 5, 785. [CrossRef]

15. Egodapitiya, K.N.; Sharma, S.; Hasan, A.; Laforge, A.C.; Madison, D.H.; Moshammer, R.; Schulz, M. Manipulating Atomic Fragmentation Processes by Controlling the Projectile Coherence. Phys. Rev. Lett. 2011, 106, 153202. [CrossRef] [PubMed]

16. Chowdhury, U.; Schulz, M.; Madison, D.H. Differential cross sections for single ionization of $\mathrm{H}_{2}$ by $75-\mathrm{keV}$ proton impact. Phys. Rev. A 2011, 83, 032712. [CrossRef]

17. Feagin, J.M.; Hargreaves, L. Loss of wave-packet coherence in stationary scattering experiments. Phys. Rev. A 2013, $88,032705$. [CrossRef]

18. Kouzakov, K.A. Theoretical analysis of the projectile and target coherence in COLTRIMS experiments on atomic ionization by fast ions. Eur. Phys. J. D 2017, 71, 70613. [CrossRef]

19. Nagy, L.; Járai-Szabó, F.; Borbély, S. The effect of projectile wave packet width on the fully differential ionization cross-sections. J. Phys. B 2018, 51, 144005. [CrossRef] 